\title{
CIERRE ESPONTÁNEO DE AGUJEROS MACULARES TRAUMÁTICOS DE ESPESOR COMPLETO
}

\section{SPONTANEOUS CLOSURE OF FULL THICKNESS TRAUMATIC MACULAR HOLES}

\author{
BOSCH-VALERO J ${ }^{1}$, MATEO J ${ }^{1}$, LAVILLA-GARCÍA L ${ }^{1}$, NÚNẼEZ-BENITO E ${ }^{1}$, \\ CRISTÓBAL JA ${ }^{2}$
}

\section{RESUMEN}

Caso clínico: Se presentan dos casos de pacientes jóvenes que sufrieron sendos agujeros maculares traumáticos de espesor completo con deterioro visual de más de un $60 \%$. Ambos mejoraron clínica y anatómicamente mientras esperaban un tratamiento quirúrgico.

Discusión: El cierre espontáneo de agujeros maculares traumáticos es un hallazgo infrecuente. El seguimiento clínico y mediante OCT permite apreciar la mejoría en las primeras semanas. Un período corto de observación nos puede evitar una intervención quirúrgica compleja y laboriosa.

Palabras clave: Agujero macular, tomografía de coherencia óptica, edema retiniano, perforación retiniana, lesión ocular.

\begin{abstract}
Case report: We present case reports of two young patients suffering from full thickness traumatic macular holes resulting in visual impairment of more than $60 \%$. Both showed anatomical and visual improvement whilst waiting for surgical treatment.

Discussion: Spontaneous closure of a traumatic macular hole is an unusual outcome. OCT and clinical follow up enabled monitoring of this resolution during a period of a few weeks. Complex surgery was thus avoided by a short observational period (Arch Soc Esp Oftalmol 2008; 83: 325-327).
\end{abstract}

Key words: Macular hole, optical coherence tomography, retinal edema, retinal perforations, eye injuries.

\section{INTRODUCCIÓN}

Los traumatismos contusos oculares se asocian a numerosas complicaciones retinianas tales como edema de Berlin, roturas periféricas y diálisis retiniana, hemorragias vítreas o subretinianas, rupturas coroideas y agujeros maculares.

A pesar de un conocimiento cada vez mejor de los mecanismos que inducen tanto a la formación como a la resolución espontánea de los agujeros maculares traumáticos (AMT) se tienen datos poco fiables respecto a la incidencia y la cronología del proceso de autosellado. En la literatura sólo se encuentran series cortas: Mizusawa et al señalan una incidencia del 10\%, Tomii et al. del 66,6\% y Yamashita et al del $44,4 \%$ pero con muestras muy escasas (10, 6, y 18 pacientes respectivamente) (1). El tiempo entre el diagnóstico y la resolución ana-

\footnotetext{
Recibido: 8/1/07. Aceptado: 14/4/08.

Hospital Clínico Universitario Lozano Blesa. Zaragoza. España.

${ }^{1}$ Licenciado en Medicina.

Correspondencia:

Jordi Bosch Valero

C/. La Pampa, 17

07003 Menorca (Baleares)

España

E-mail: jordiperebosch@yahoo.es
} 
tómica del AMT también es variable, entre 1 semana y seis meses según las publicaciones.

Se presentan los casos de dos pacientes con AMT tras contusión ocular, bien documentados mediante tomografía de coherencia óptica (OCT), en los que se produjo un cierre espontáneo del mismo en menos de 6 semanas con una mejora visual significativa.

\section{CASO CLÍNICO}

\section{Caso 1}

Varón de 36 años que acudió a nuestro servicio en febrero 2006 seis días después de haber sufrido una contusión con un clavo en el ojo izquierdo (OI). Refería deterioro visual inmediato con escotoma central del mismo ojo. A la exploración, se observó una agudeza visual (AV) en el OI de 0,4 y en el ojo derecho (OD) de la unidad. Mediante la rejilla de Amsler se manifestaba una alteración central y metamorfopsias en el OI. En la biomicroscopía se apreciaba una iritis moderada y en el fondo de ojo, hemovítreo inferior, hemorragia peripapilar y un agujero macular de espesor completo (fig. 1). Test de Watkze-Allen, positivo. La OCT confirmó la presencia de un agujero macular de espesor com-

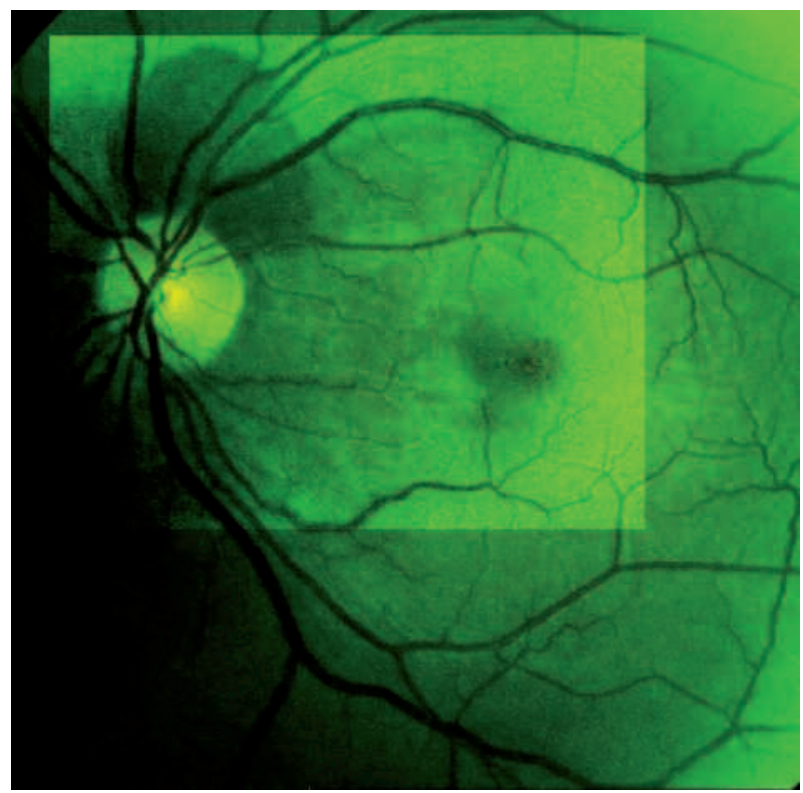

Fig. 1: Retinografía con filtro verde en la que se aprecia una hemorragia suprapapilar y un agujero macular traumático.

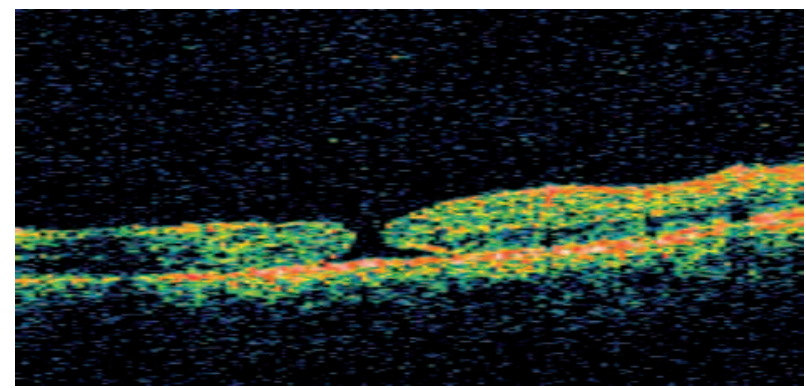

Fig. 2: OCT en el que se observa el agujero macular de espesor completo.

pleto (fig. 2) en el OI por lo que se programó para cirugía (vitrectomía vía pars plana y extracción de la membrana limitante interna) y se instauró tratamiento con Prednisona oral a dosis de $1 \mathrm{mg} / \mathrm{kg} / \mathrm{día}$ en pauta descendente, junto con protección gástrica. El día previo a la cirugía (35 días después del traumatismo) el paciente se exploró de nuevo constatándose una mejoría evidente de la agudeza visual con el OI y un cierre completo de la lesión demostrada por la OCT (fig. 3).

\section{Caso 2}

Varón de 16 años que acude a nuestro servicio de oftalmología en abril del 2006 por pérdida de agudeza visual y escotoma central tras impacto ocular en OI con un balón de fútbol. La AV era de contar dedos a 2 m en el OI y de la unidad en el contralateral. A la exploración del fondo de ojo se apreció edema de Berlin macular de predominio suprafoveolar e imagen de agujero macular completo (fig. 4) que fue confirmada por la OCT (fig. 5). La rejilla de Amsler y el test de Watzke-Allen aportaron resultados congruentes. El tratamiento fue idéntico al del caso antes descrito. A las dos semanas el paciente

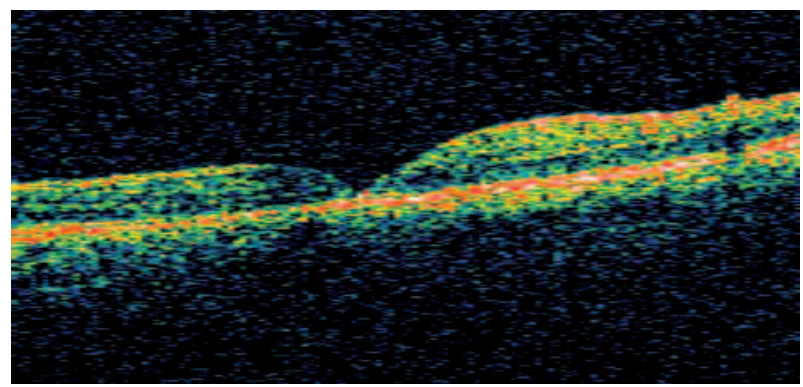

Fig. 3: OCT del mismo paciente 42 días después en la que se aprecia resolución completa de la lesión. 


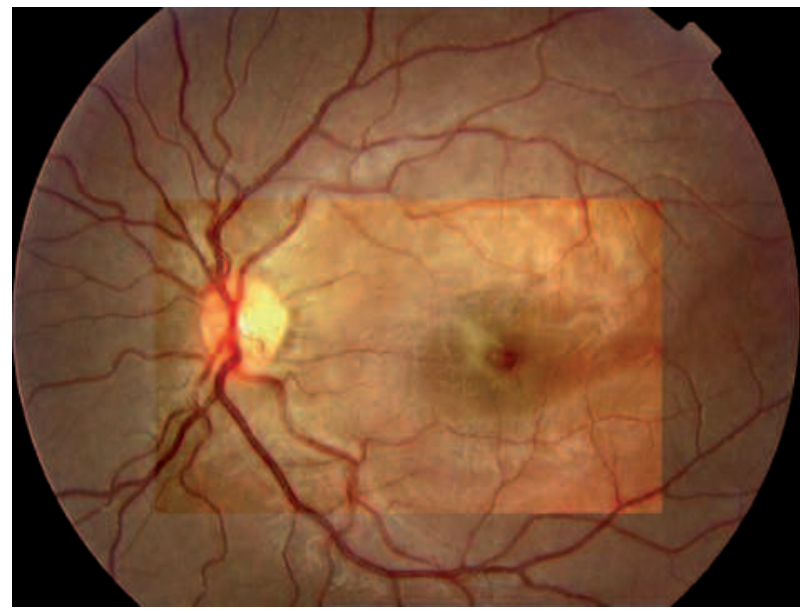

Fig. 4: Edema del polo posterior y agujero macular traumático del paciente $n .{ }^{\circ} 2$.

acudió a revisión y se constató una mejoría del OI, con una agudeza visual de 0,3 y un autosellado de la lesión demostrado por OCT (fig. 6).

\section{DISCUSIÓN}

Los casos de AMT autosellado que han sido publicados hasta la fecha coinciden en dos puntos: en primer lugar son todos pacientes de menos de 20 años $\mathrm{y}$, en segundo lugar, en todos los casos la lesión es consecuencia de un traumatismo contuso. Uno de nuestros pacientes supera ampliamente esa edad, con 36 años.

El deterioro de la agudeza visual puede ser tanto inmediato como retardado por lo que Yamashita propuso dos mecanismos de formación: la dehiscencia primaria de la fóvea inmediata al traumatismo y la persistencia de una tracción vítrea que origina el agujero macular días después (1). En nues-

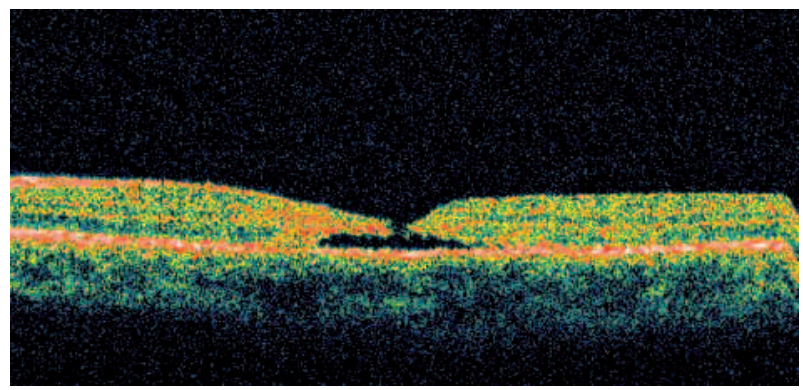

Fig. 5: Agujero macular completo con adelgazamiento de los bordes y líquido subretiniano.

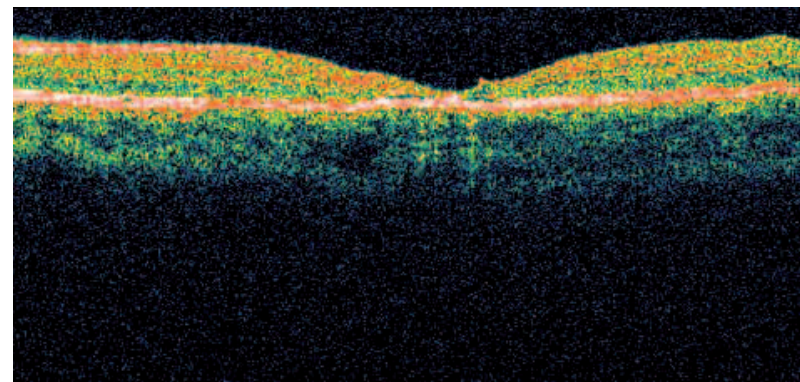

Fig. 6: OCT del paciente n. $^{\circ} 20$ días después en la que se aprecia una resolución completa.

tros dos pacientes la clínica fue inmediata y no se observaron tracciones vítreas en la OCT.

El tiempo transcurrido desde el traumatismo al cierre espontáneo es variable según las distintas publicaciones. El caso publicado por Yeshurun et al. tardó 5 meses (2), los tres de Kusaka et al. entre 3 y 4 meses (3), los de Yamada et al. entre 4 y 6 (4). Yamashita et al. presentaron en el año 2002 ocho casos que tardaron entre 1 semana y 4 meses es cerrar, citando a Mizusawa (9 meses), a Tomii (14 días a 5 meses), Nunode ( 15 días), Murakami (3 meses) y Parmar (2 meses) (1). Lai et al. Publicaron en el año 2006 el caso de un AMT con desprendimiento de retina secundario que tardó tres semanas en autosellarse (5).

En nuestros dos casos el cierre fue precoz (42 y 20 días respectivamente) mientras se planteaba la cirugía. A pesar de los buenos resultados obtenidos por las técnicas quirúrgicas actuales, consideramos importante esperar unas semanas antes de indicar la cirugía en pacientes jóvenes con AM traumático por la posibilidad del cierre espontáneo de los mismos en este subgrupo de pacientes.

\section{BIBLIOGRAFÍA}

1. Yamashita T, Uemara A, Uchino E, Doi N, Ohba N. Spontaneous closure of traumatic macular hole. Am J Ophthalmol 2002; 133: 230-235.

2. Yeshurun I, Guerrero-Naranjo JL, Quiroz-Mercado $H$. Spontaneous closure of a large traumatic macular hole in a young patient. Am J Ophthalmol 2002; 134: 602-603.

3. Kusaka S, Fujikado T, Ikeda T, Tano Y. Spontaneous disappearance of traumatic macular holes in young pacients. Am J Ophthalmol 1997; 123: 837-839.

4. Yamada H, Sakai A, Yamada E, Nishimura T, Matsumura M. Spontaneous closure of traumatic macular hole. Am J Ophthalmol 2002; 134: 340-347.

5. Lai MM, Joshi MM, Trese MT. Spontaneous resolution of traumatic macular hole-related retinal detachment. Am J Ophthalmol 2006; 141: 1148-1151. 